Trauma Berufskrankh 2006 • 8[Suppl 2]: S155-S160 DOI 10.1007/s10039-005-1019-5

Online publiziert: 20. Juli 2005

๑) Springer Medizin Verlag 2005

M. Braun · E. Kiesouw · BVB 09, Dortmund

\title{
Kapsel-Band-Verletzung des Kniegelenks
}

\section{Nachbehandlungsalgorithmen in Abhängigkeit vom operativen Vorgehen und der Hilfsmittelversorgung}

Spezielle Ziele der Rehabilitation von Knieverletzungen sind das Erreichen des Niveaus:

- Der Beweglichkeit,

- Der Kraft,

- Der Schnelligkeit und

- Der Koordination entsprechend der gesunden Gegenseite;

- Des Laufvermögens und

- Der arbeits- bzw. sportspezifischen Belastbarkeit entsprechend dem Niveau vor der Verletzung.

Schwere Kapsel-Band-Verletzungen des Kniegelenks spielen ökonomisch und medizinisch eine große Rolle. In der Profifußballabteilung von Borussia Dortmund traten zwischen Sommer 1998 und Sommer 2004 insgesamt 18 dieser Verletzungen auf. Das entspricht 1/3 aller schweren Gelenkverletzungen.
Die Mindestausfallzeiten der verletzten Spieler beliefen sich auf 3-6 Monate. Sowohl für den Betroffenen als auch für den Verein haben diese langen Fehlzeiten erhebliche finanzielle und sportliche Verluste zur Folge. Zusätzlich ist der medizinische Aufwand mit Diagnostik, Erstbehandlung, evtl. Operation, Orthese, Rehabilitation und nach Wiederaufnahme des Trainings- und Wettkampfbetriebs begleitender Rezidivprävention sehr groß.

In diesem Handlungsfeld gibt es viele praktisch orientierte Hinweise, der Stand der wissenschaftlichen Fundierung ist jedoch noch als mangelhaft einzustufen [4].

Ziel dieser Arbeit ist die Vorstellung eines algorithmisch aufgebauten Rehabilitationsbaums, der eine Beurteilung der Leistungsfähigkeit des Verletzten während und der Sportfähigkeit am Ende der Rehabilitation erlaubt.
- Prävention von Rezidiven und Folgeschäden.

\footnotetext{
Abb. $1>$ Orthesen für a Innen- und vordere Kreuzbandrehabilitation, b hintere Kreuzbandrehabilitation
}

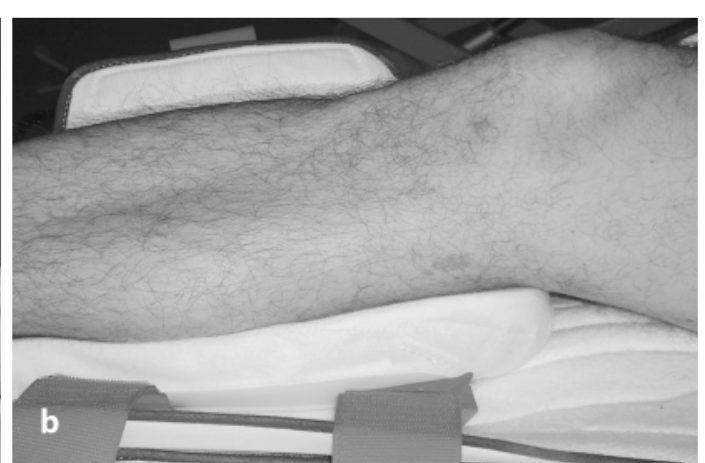


Kniegelenk

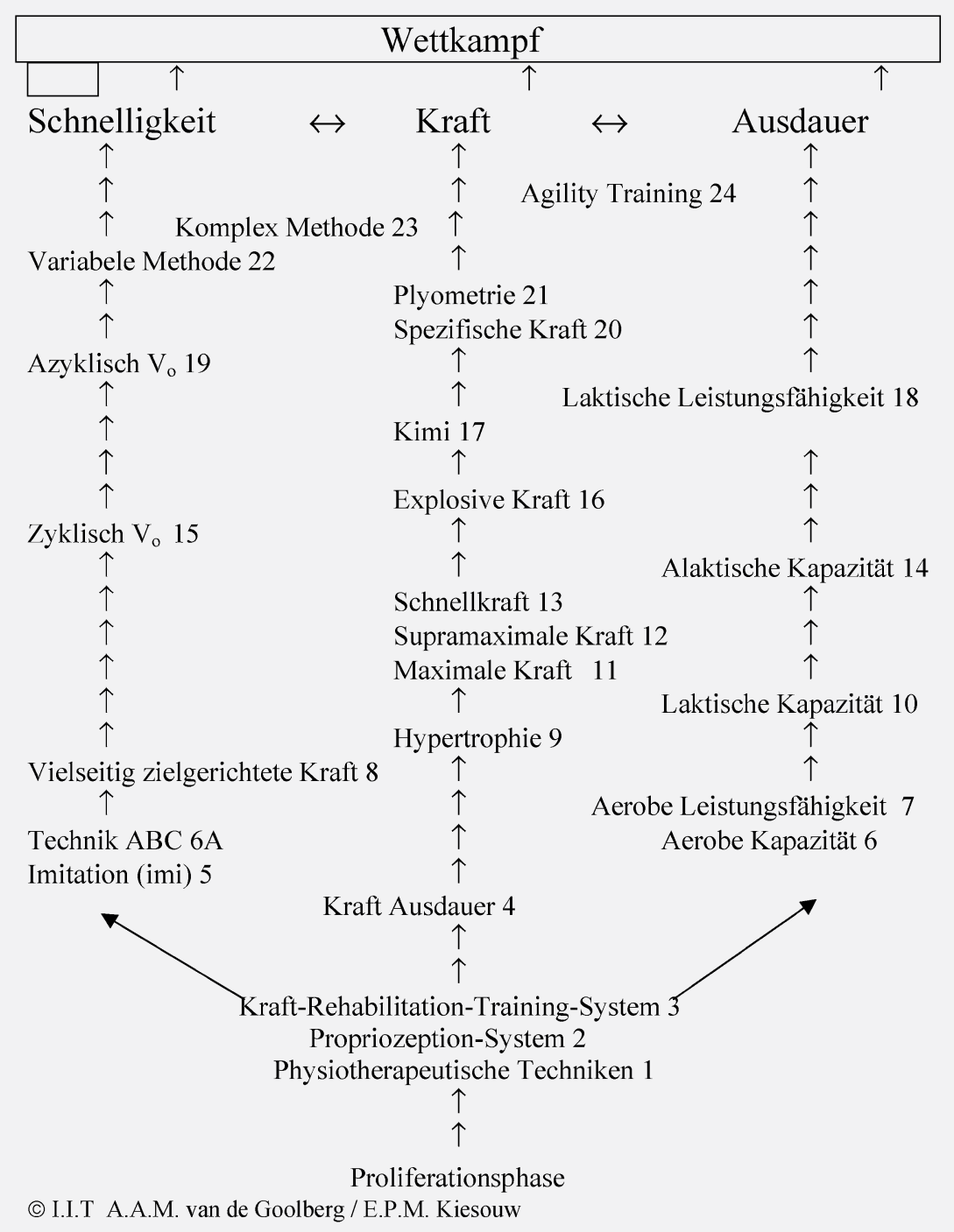

Abb. $2 \Delta$ Rehabilitationsbaum mit den Hauptästen Schnelligkeit, Kraft und Ausdauer, die in viele Einzelbausteine zerlegt sind, die jeweils eine Rehabilitationseinheit darstellen

\section{Material und Methode}

Der erste Schritt der Rehabilitation ist ein theoretischer: die Analyse der Verletzungsursachen. Als mögliche Gründe für Kapsel-Band-Verletzungen des Kniegelenks sind zu nennen:

1. Es besteht ein allgemein hohes Risiko, Gelenkverletzungen bei Kontaktsportarten, z. B. Fußball, zu erleiden.

2. Profisportler haben häufig in ihrer Anamnese Vorverletzungen der Kniegelenke einschließlich Voroperationen mit verbleibenden Defiziten.

3. Die Qualität des Kapsel-Band-Apparats ist mangelhaft (z. B. propriozeptive Fähigkeit, Trophik der Bänder).
4. Die Qualität der aktiven, Gelenk stabilisierenden Muskulatur und Sehnen ist mangelhaft (z. B. neuromuskuläre Reaktionszeit, Kraftfähigkeiten).

5. Muskuloskelettale Formvarianten (z. B. Genua valga)

6. Zu starke Fixierung des Fußes am Boden in Abhängigkeit von der Besohlung des Schuhs und der Beschaffenheit des Untergrunds.

7. Mangelhafte Aufmerksamkeit und Konzentration während der Arbeit oder des Sports.

8. Fehlende Antizipation von Risikosituationen, langsame geistige und körperliche Reaktionszeit, fehlende reaktive Körperspannung.
9. Allgemeine Ermüdung von Profisportlern aufgrund hoher physischer und psychischer Belastung über einen Zeitraum von mehreren Jahren mit vielfachen nationalen und internationalen Wettbewerben, geringe Rotation in einem Team bei gleich bleibendem Kader über Jahre.

10. Trainingsbedingungen und Trainingsinhalte:

- Zu wenig präventive sportartspezifische Belastungssituationen

- Zu wenig spezifisches Präventionstraining bei ausgeruhten, aufnahmefähigen Sportlern

- Zu häufig monotone Ausdauerbelastung

11. Häufiger Wechsel der Vereine und des medizinischen Personals im Profisport mit den Folgen:

- Fehlende medizinische Kontinuität in der Analyse von Defiziten des Kapsel-Band-Apparats und der Verletzungsprävention

- Niedriger Stellenwert und geringe Einflussnahme der Mediziner auf Trainingsinhalte

Diese und weitere allgemeine sowie individuelle Verletzungsursachen sind bei jeder Verletzung des Kniegelenks zu berücksichtigen und müssen nach Wertigkeit für den Betroffenen Würdigung in dem individuellen Rehabilitationsplan finden.

Die Rehabilitation orientiert sich in der Regel an der Wundheilung, welche sich in 4 Phasen gliedert $[7,12]$ :

- Zunächst tritt unmittelbar nach dem Trauma bzw. der Operation die Entzündungsphase ein. Diese dauert etwa 5 Tage und ist durch die Einwanderung von Entzündungszellen in die verletzte Region sowie Gefäßeinsprossung charakterisiert.

- Danach folgt bis etwa zum 21. Tag die Proliferationsphase, welche durch die Synthese von Kollagen Typ III charakterisiert ist.

- Daran schließt sich die Konsolidierungsphase bis etwa zum 6o. Tag mit der Synthese von Kollagenfasern Typ I an.

- Dann folgt die Remodellierungsphase bis etwa 360 Tage nach der Verletzung, wobei sich das Gewebe in Ab- 
hängigkeit von Umgebungsreizen anpasst und eine vollständige Regeneration eintreten kann.

Als Grundregeln der Rehabilitation sind zu berücksichtigen:

- Training und die direkte Erholung dauern jeweils Minuten bis Stunden.

- Für die wirkliche Anpassung des Gewebes auf einen Trainingsreiz im Sinn einer Superkompensation benötigt der Körper Stunden bis Tage [12].

Diese Regeln sollten im Rahmen der Rehabilitation unbedingt berücksichtigt werden, da zu kurze Erholungsphasen zu einer relativen Überlastung und somit Verschlechterung der Regeneration führen. Dies hat eine Verlängerung der Wundheilungsphasen und der Rehabilitation mit dem erhöhten Risiko eines Verletzungsrezidivs zur Folge.

In Abhängigkeit vom Verletzungsmuster und nach Vorgabe des Operateurs kann das Tragen einer Orthese für die Verrichtungen des täglichen Lebens für meist 6 Wochen erforderlich sein (• Abb. 1).

In der Literatur wird die Notwendigkeit des Tragens einer Kniegelenkschiene nach vorderer Kreuzbandplastik kontrovers diskutiert. Randomisierte, prospektive Studien zeigten keine signifikanten Unterschiede in Funktion und Stabilität bei Nachuntersuchungen bis zu 2 Jahren nach vorderer Kreuzbandplastik mit oder ohne Orthesennachbehandlung $[1,8,9,11]$. Andererseits wurde ein Effekt auf die propriozeptiven Fähigkeiten der betroffenen Muskulatur berichtet [10].

Nachfolgend werden Nachbehandlungsalgorithmen für eine systematische Rehabilitation nach Kiesouw [6] und Goolberg u. Kiesouw [5] vorgestellt. Sie haben ein Rehabilitationsschema entwickelt (- Abb. 2), welches die Grundvoraussetzungen klar analysiert, die am Ende einer Rehabilitation optimal ausgebildet sein müssen. Diese Voraussetzungen für Wettkampf- bzw. Arbeitsfähigkeit sind

- Schnelligkeit,

- Kraft und

- Ausdauer

Diese Grundpfeiler der körperlichen Leistungsfähigkeit wurden von Kiesouw [6]

Trauma Berufskrankh 2006 $\cdot 8$ [Suppl 2]: S155-S160

DOI 10.1007/s10039-005-1019-5

(c) Springer Medizin Verlag 2005

M. Braun · E. Kiesouw

Kapsel-Band-Verletzung des Kniegelenks. Nachbehandlungsalgorithmen in Abhängigkeit vom operativen Vorgehen und der Hilfsmittelversorgung

\section{Zusammenfassung}

Der erste Schritt der Rehabilitation ist ein theoretischer: die Analyse der Verletzungsursachen. Diese müssen im individuellen Rehabilitationsplan berücksichtigt werden, der sich an den Wundheilungsphasen orientiert. Der vorgestellte Rehabilitationsbaum analysiert die Grundvoraussetzungen, die am Ende der Maßnahme optimal ausgebildet sein müssen. Voraussetzungen für Wettkampf- bzw. Arbeitsfähigkeit sind Schnelligkeit, Kraft und Ausdauer. Alle Einzelkomponenten des Rehabilitationsbaums bauen algorithmisch aufeinander auf. Sollte eine Leistungsstufe noch nicht erreicht sein, findet auch kein Übergang zu höheren Trainingsstufen statt. Zur Steuerung des Heilverfahrens erfolgen die orthopädische klinisch-dynamische Leistungsdiagnostik mit Bewertungen des Bewegungsausmaßes und der Kraftausdauer-

fähigkeit an der Langhantel sowie in späteren Rehabilitationsphasen die Analyse der Sprungfähigkeit. Sie erlauben die Beurteilung der Leistungsfähigkeit des Verletzten während der Rehabilitation und der Sportfähigkeit am Ende derselben. Die dynamische Leistungsdiagnostik sollte in wissenschaftlichen Studien untersucht werden, um einen allgemein anerkannten Standard an Messmethoden zu evaluieren. Ein solcher ist auch zur Prävention zu erarbeiten, um individuelle Risikofaktoren erkennen und wissenschaftlich überprüfbare Präventionsprogramme entwickeln zu können.

\section{Schlüsselwörter \\ Kapsel-Band-Verletzungen Kniegelenk . Verletzungsursachen · Rehabilitation · Orthopädische dynamische Leistungsdiagnostik · Prävention}

\section{Capsular ligament injuries of the knee. Follow-up algorithms dependent on surgical procedures and rehabilitation}

\section{Abstract}

The first step in rehabilitation is to analyze of the cause of the trauma. A rehabilitation schedule that is oriented to individual wound healing stages must be used. The rehabilitation-tree analyses the basic physical qualities that have to be established and optimized at the end of rehabilitation. Speed, strength and endurance are the essential requirements for athletic or physical performance. These individual components are assembled algothithmically in the rehabilitation-tree. If a defined level of performance is not reached, rehabilitation will not proceeded to the following level. In order to control rehabilitation, orthopedic, clinical-dynamic performance diagnostics are used. These include evaluation of mobility and strengthendurance ability using barbells and, at a later stage, the evaluation of jumping ability.
Such examinations can be used to evaluate the general physical performance of traumatized athletes during rehabilitation and their athletic ability at the end of rehabilitation. Controlled clinical trials are necessary to assess dynamic performance diagnostics so that standard methods can be evaluated. Standardized dynamic performance diagnostics should also be developed to aid prevention by identifying individual risk factors and further develop scientifically testable prevention programs.

\section{Keywords}

Capsular ligament injuries of the knee - Cause of trauma - Rehabilitation . Orthopedic dynamic performance diagnostics · Prevention 


\section{Kniegelenk}
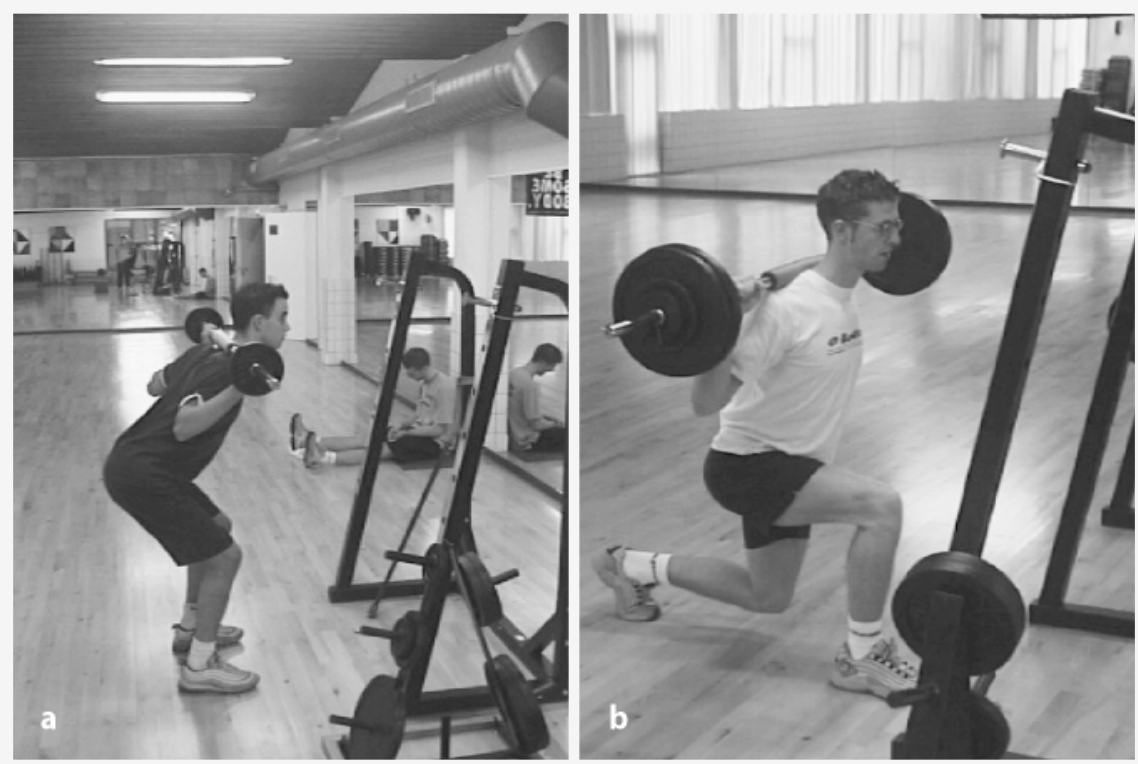

Abb. $3<$ a Beginn, b erreichtes

Ziel des Krafttrainings in der geschlossenen Kette, gleichzeitig Bewertung von Bewegungsausmaß und Kraftausdauerfähigkeit

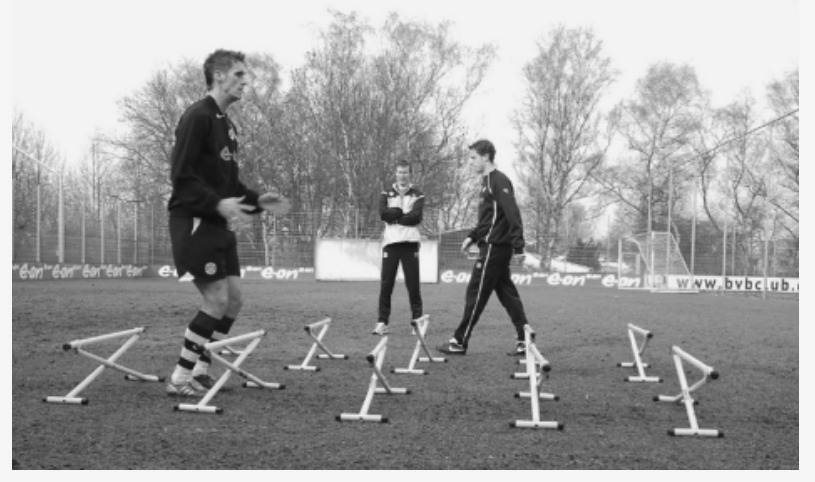

Abb. $4<$ Sprungtraining und Bewertung der Sprungfähigkeit und Goolberg u. Kiesouw [5] in viele Einzelbausteine zerlegt, die jeweils eine Rehabilitationseinheit darstellen. In • Tabelle 1 findet sich beispielhaft der Inhalt für eine Übungseinheit der spezifischen Kraft zum Ende einer Rehabilitation in Form von kurzen horizontalen Sprüngen. Die Inhalte eines Trainings sind eindeutig definiert und werden vom Verletzten exakt protokolliert, um eine eigenverantwortliche Kontrolle der Entwicklung zu haben.

Alle Einzelkomponenten des Rehabilitationsbaums (- Abb. 2) bauen algorithmisch aufeinander auf, sowohl in vertikaler Richtung als auch zwischen den Grundpfeilern Schnelligkeit, Kraft und Ausdauer in horizontaler Richtung. In Abhängigkeit vom Anforderungsprofil des Sportlers bzw. Arbeiters werden klare Ziele einer jeden Rehabilitationseinheit definiert. Sollte eine Leistungsstufe noch nicht erreicht sein, findet auch kein Übergang zu höheren Trainingsstufen statt. Dadurch läuft man keine Gefahr, Defizite am Ende einer Rehabilitation zu übersehen, den Rehabilitierten zu gefährden und mühsam fehlende Komponenten aufholen zu müssen.

Beispielhaft wird die spezifische Rehabilitation nach operativer Rekonstruktion des vorderen Kreuzbands nach Goolberg u. Kiesouw [5] vorgestellt, welche sich an den Phasen der Wundheilung orientiert. In Abhängigkeit von der Transplantatentnahmestelle (Patellarsehne, Semitendinosussehne) muss ein propriozeptives Defizitsyndrom des M. quadriceps oder der ischiokruralen Muskulatur insbesondere innerhalb der ersten 6 Wochen berücksichtigt werden.

Grundsätzliche Maßnahme vor jeder Therapiesitzung ist die physiotherapeutische Kontrolle folgender klinischer Befunde:

$$
\begin{aligned}
& \text { - Schmerz } \\
& \text { - Erguss } \\
& \text { - Bewegungsausmaß }
\end{aligned}
$$

- Kniescheibenmobilität
- Muskelkontrolle
- Gangbild

- Tabelle 2 zeigt das Beispiel einer frühen Rehabilitationsphase mit der klaren Gliederung in allgemeine Bemerkungen, Grundvoraussetzungen, Rehabilitationsinhalte und Ziele, die erreicht werden müssen, um in die nächste Rehabilitationsphase wechseln zu können. Die kompletten Rehabilitationsprotokolle können beim Autor angefordert werden.

Zur Steuerung des Heilverfahrens ist die klinische Leistungsdiagnostik mit Bewertungen des Bewegungsausmaßes und der Kraftausdauerfähigkeit möglich ( $\bullet$ Tabelle 3, Abb. 3). In der Remodellierungsphase ist dann als zusätzliches Kriterium die Sprungfähigkeit als klinischer Parameter wertvoll (• Abb. 4).

In der apparativen Leistungsdiagnostik existieren eine Vielzahl von Geräten, welche in bestimmten Einzelfällen sinnvoll 
Tabelle 1

Übungseinheit der spezifischen

Kraft zum Ende einer Rehabilita-

tion mit gleichzeitigem Protokoll

Test horizontale kurze Sprünge

Datum

Name

\begin{tabular}{|l|}
\hline $\begin{array}{l}\text { Übung } \\
\text { 3-Sprung aus Stand }\end{array}$ \\
\hline 3-Hüpf links \\
\hline 3-Hüpf rechts \\
\hline 5-Sprung \\
\hline 5-Hüpf links \\
\hline 5-Hüpf rechts \\
\hline 10-Sprung \\
\hline 10-Hüpf links \\
\hline 10-Hüpf rechts \\
\hline
\end{tabular}

Aus Goolberg u. Kiesouw [5]

eingesetzt werden können. Beispielhaft sind zu nennen:

- KT 1000

- Videolaufanalyse [2]

- Dreidimensionale Bewegungsanalyse [2]

- Propriozeptionstest [2]

- Isokinetiktest

- Sprunganalyse

- Winkelreproduktionstest

- Fastex

- CAT 2000

Zeigen sich in der Leistungsdiagnostik am Ende der Rehabilitation des Spitzensportlers

- eine freie Kniegelenkbeweglichkeit,

- Koordinationsvermögen, Kraft- und Propriozeptionsfähigkeit entsprechend dem Niveau der gesunden Extremität,

- Laufvermögen entsprechend dem Niveau vor der Verletzung,

- ausreichendes Selbstvertrauen und Motivation des Sportlers bzw. Arbeiters,

sind die speziellen Rehabilitationsziele erreicht, und eine Wiederaufnahme des Wettkampfbetriebs bzw. der körperlichen Arbeit ist möglich.

Tabelle 2

Protokoll einer frühen Phase der spezifischen Rehabilitation nach operativer Rekonstruktion des vorderen Kreuzbands

Rehabilitationsprotokoll: Wochen 5-6

Allgemeine Bemerkungen

- Vollbelastung (kein Schongang!)

- Hämarthroskontrolle

- Postoperative Schmerzkontrolle ohne NSAID

- Muskelkontrolle in $0^{\circ}$ Extension erreichen

- ROM (E/F)

$0 / 0 / 120^{\circ}$

Grundvoraussetzung

- Schmerz Erträglich

- Hämarthros Minimal

- Patellamobilisation Gut

- Minimaler ROM (E/F) 0/0/120

- Muskelkontrolle Gut

Rehabilitation

- ROM

- Flexion

- Kraft

"Betonung der Extension"

Durch geschlossene Kette, Anfersen

Q-Kontraktion $0^{\circ}$-Position, geschlossene Kette 0-60

- Gehschule

- Koordination

- Ausdauervermögen „Betonung der Extension", ,",cup walking" Wackelbrett Crosswalker, Fahrrad (kurze Kurbel), Spazierengehen

Ziele

- ROM (E/F)

0/0/120-135

- Volle Muskelkontraktion, Kraftausdauer

- Vollbelastung

- Vorhersehen von Komplikationen

Tabelle 3

Klinisch-dynamische Leistungsdiagnostik

\begin{tabular}{|c|c|c|}
\hline Wundheilungsphasen & Bewegungsausmaß & Kraft, Koordination (,squats“) \\
\hline Entzündung (1.-5. Tag) & F/E $90 / 0 / 0^{\circ}$ & \\
\hline Proliferation (bis 21. Tag) & F/E $120-135 / 0 / 0^{\circ}$ & GK $30-45 / 0^{\circ}$ \\
\hline Konsolidierung (bis 60. Tag) & $\mathrm{F} / \mathrm{E} 140 / 0 / 0^{\circ}$ & GK $60 / 0^{\circ}$ \\
\hline Remodellierung ( $90-360$ Tage) & $\mathrm{F} / \mathrm{E} 140 / 0 / 0^{\circ}$ & GK $90 / 0^{\circ}$ \\
\hline
\end{tabular}

In der Regel bedeutet dies eine Wiederaufnahme des Sports nach vorderer Kreuzbandplastik:

- Für Geradeauslaufsportarten nach Abschluss des 3. postoperativen Monats,

- Bei Kontaktsportarten nach Abschluss des 6. postoperativen Monats.

Die Wiederaufnahme der Arbeit oder des Wettkampfbetriebs ist jedoch grundsätz- lich individuell $\mathrm{zu}$ entscheiden und insbesondere abhängig von Begleitverletzungen, die eine Belastungsfähigkeit beeinflussen:

- Innen-, Außenbandverletzung, Instabilität der posterolateralen Ecke

- Meniskusverlust

- Knorpelschaden 


\section{Fazit für die Praxis}

\section{Zur Beurteilung der Leistungsfähigkeit} des Verletzten während der Rehabilitation und der Sportfähigkeit am Ende der Rehabilitation sind wissenschaftliche Studien zur Überprüfung der beschriebenen Methoden zur Erhebung dynamischer Leistungsparameter erforderlich. Basierend auf den aus solchen Studien gewonnenen wissenschaftlichen Daten ist die Festlegung eines allgemein anerkannten Standards wiederholbarer, phasenangepasster und kostengünstiger Messmethoden notwendig, um die optimale Steuerung eines Heilverfahrens zu gewährleisten.

Ein solcher Standard mit gleicher dynamischer Leistungsdiagnostik ist auch zur Prävention zu erarbeiten, um individuelle Risikofaktoren zu erkennen und wissenschaftlich überprüfbare Präventionsprogramme zu entwickeln.

\section{Korrespondierender Autor}

\section{Dr. M. Braun}

BVB 09,

Rheinlanddamm 207-209, 44137 Dortmund

E-Mail: braun@microtherapy.de

Interessenkonflikt: Keine Angaben

\section{Literatur}

1. Brandsson S, Faxen E, Kartus J et al. (2001) Is a knee brace advantageous after anterior cruciate ligament surgery? A prospective, randomised study with a two year follow-up. Scand J Med Sci Sports 11: 110-114

2. Braun M, Kuhnt P, Zöllner F et al. (2004) Kapselbandverletzungen des Kniegelenkes beim Leistungssportler. Spezifische Rehabilitationsmaßnahmen beim Spitzensportler. 18. Duisburger Gutachtenkolloquium - Chirurgischer Teil. Duisburg 19.20.11.2004

3. Ellenbecker TS (2000) Knee ligament rehabilitation. Churchill Livingstone, Edinburgh London New York

4. Freiwald J, Engelhardt N (2002) Orthopädisch/Traumatologische Rehabilitation, Prävention und Rehabilitation, Stand des motorischen Lernens und der Koordination in der orthopädisch/traumatologischen Rehabilitation. Sportorthop Sporttraumatol 18: 5-10

5. Goolberg van de AAM, Kiesouw E (2001) Cursus topsport. Arnhem

6. Kiesouw E (2003) Kruisbandreconstructieve ingrepen zijn leuk. Maar, Maasland Ziekenhuis

7. Maibaum S, Braun M, Jagomast B et al. (2001) Therapielexikon der Sportmedizin. Springer, Berlin Heidelberg New York
8. McDevitt ER, Taylor DC, Miller MD et al. (2004) Functional bracing after anterior cruciate ligament reconstruction: a prospective, randomized, multicenter study. Am J Sports Med 32: 1887-1892

9. Moller E, Forssblad M, Hansson L et al. (2001) Bracing versus nonbracing in rehabilitation after anterior cruciate ligament reconstruction: a randomized prospective study with 2-year follow-up. Knee Surg Sports Traumatol Arthrosc 9: 102-108

10. Ramsey DK, Wretenberg PF, Lamontagne M et al. (2003) Electromyographic and biomechanic analysis of anterior cruciate ligament deficiency and functional knee bracing. Clin Biomech 18: 28-34

11. Risberg MA, Holm I, Steen H et al. (1999) The effect of knee bracing after anterior cruciate ligament reconstruction. A prospective, randomized study with two years' follow-up. Am J Sports Med 27: 76-83

12. Van Wingerden BAM (1998) Bindegewebe in der Rehabilitation. Scipro, Schaan, Liechtenstein

13. Walla DJ, Albright JP, McAuley E et al. (1985) Hamstring control and the unstable anterior cruciate ligament-deficient knee. Am J Sports Med 13: 3439

14. Yack HJ, Riley LM, Whieldon TR (1993) Anterior tibial translation during progressive loading of the ACL-deficient knee during weight-bearing and nonweight-bearing isometric exersice. J Orthop Sports Phys Ther 20: 247-253 